\title{
Increasing follicular resistance to bacterial contamination: A novel antimicrobial therapy?
}

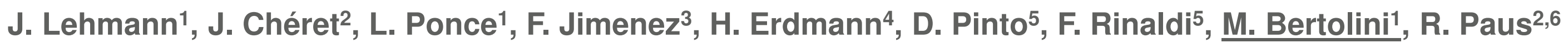

in

Monasterium Laboratory

MM Skin \& Hair Research Solutions

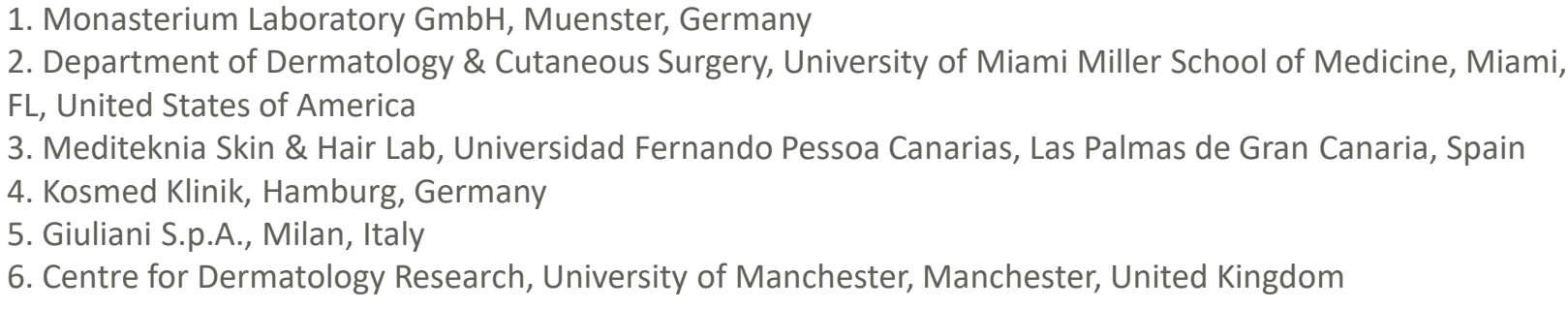

E-mail: j.lehmann@monasteriumlab.com

\section{BACKGROUND}

Olfactory receptors (OR) are part of an evolutionarily ancient chemosensory signaling system regulating many cell functions beyond olfaction. We have shown that human hair follicle (HF) epithelium engages in OR-dependent chemosensation via the OR2AT4 receptor and that continuous signaling through this receptor is required for anagen maintenance ex vivo. Intriguingly, microarray analysis revealed that Sandalore ${ }^{\circledR}$ strongly up-regulates transcription of dermcidin (DCD), an antimicrobial peptide, in microdissected HFs ex vivo ${ }^{1}$.

\section{AIM}

We asked whether Sandalore ${ }^{\circledR}$ I) actually upregulates intrafollicular DCD peptide production, and II) renders organ-cultured human HFs less susceptible to bacterial contamination/infection.

\section{EXPERIMENTAL PROCEDURE}

Amputated or full-length HFs were microdissected from occipital scalp skin or follicular unit explants and cultured in William's E complete medium only, or stimulated with Sandalore ${ }^{\circledR}$ $(500 \mu \mathrm{M})$, for 6 days $^{2}$. For the contamination experiment both groups were kept with or without antibiotics, respectively. In human scalp skin ex vivo, Sandalore ${ }^{\circledR}$ was applied topically (using PEG6000) or systemically into the medium every other day ${ }^{3}$

\section{RESULTS}

1) DCD RNA can be found in the HF epithelium, while protein is nearly undetectable, in human skin in vivo

RNA in situ hybridization

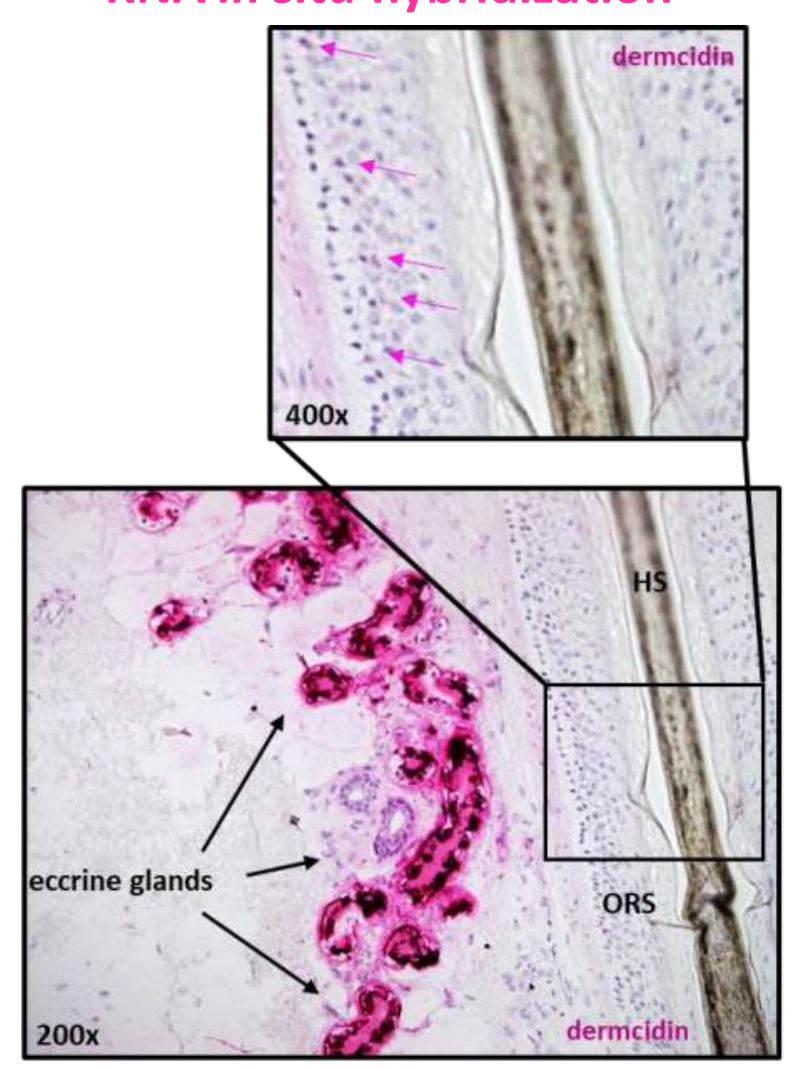

Qualitative observation from one biopsy/donor immediately frozen after collection from $n=3$ healthy donors, using a probe specifically designed for human dermcidin DCD expression has been reported in eccrine and seballow star = excetory dut of sweat glo therefore we applied this as internal positive control ${ }^{4}$.

4) Topical Sandalore ${ }^{\circledR}$ treatment tendentially increases dermcidin secretion in the HF outer root sheath in skin ex vivo

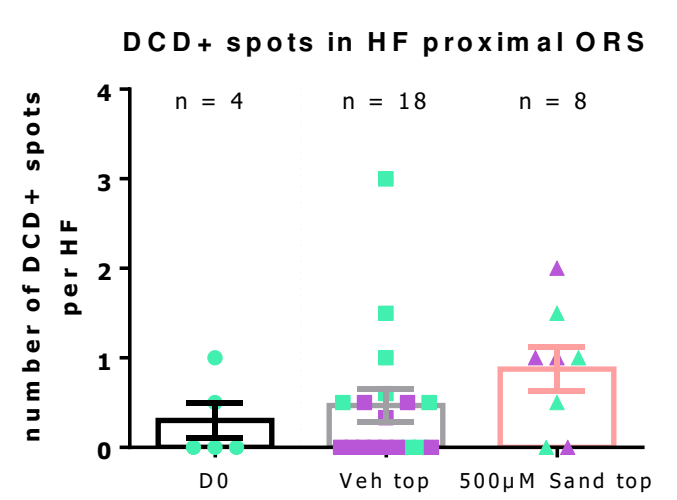

$\mathrm{n}=2$ punches from one healthy donor, 2-9 HF/group; mean \pm SEM GraphPad Prism 6; DCD spots were counted in the HF epithelium.

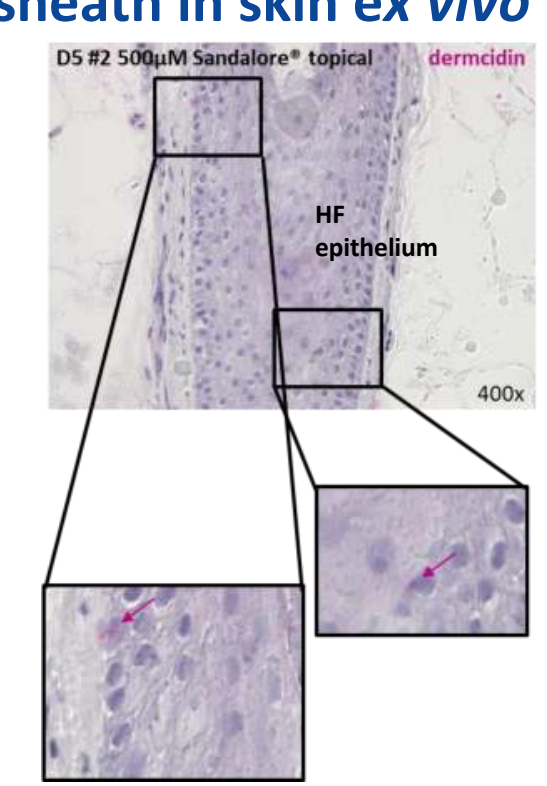

These data confirmed the results of our pilot study only using $500 \mu \mathrm{M}$ topically applied Sandalore ${ }^{\circledR}$ (data not shown)

5) Culture medium of Sandalore ${ }^{\circledR}$ treated scalp skin affects microbial balancing and influences microbial communication

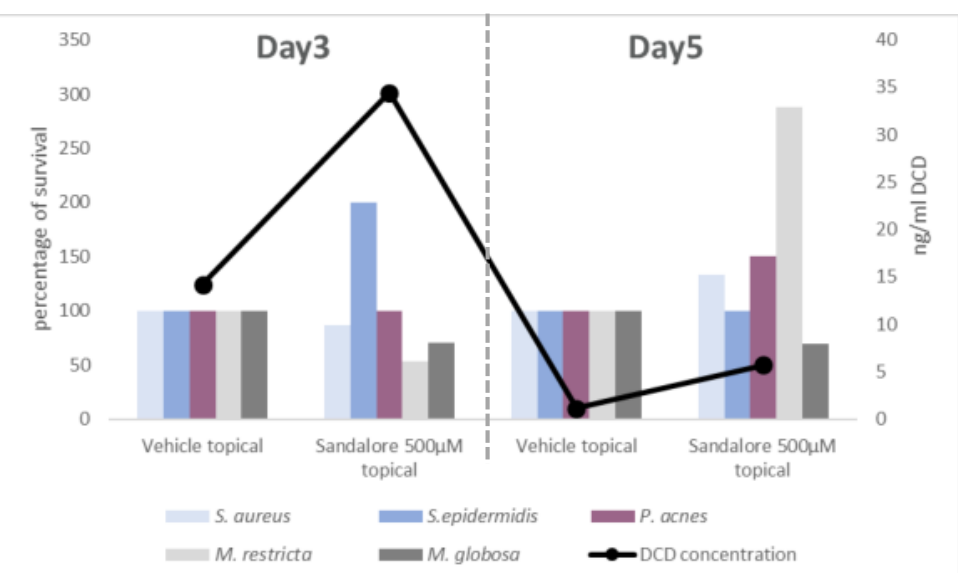

Preliminary data from $n=2$ punches of one healthy donor (see above) on day 3 and 5 of organ-culture.

Different bacterial and fungal strains were incubated with the culture medium and survival was quantified using an MTT assay. The data show the strain survival compared with the DCD concentration in the medium.
2) Sandalore ${ }^{\circledR}$ treatment up-regulates intrafollicular DCD peptide production in the HF epithelium ex vivo

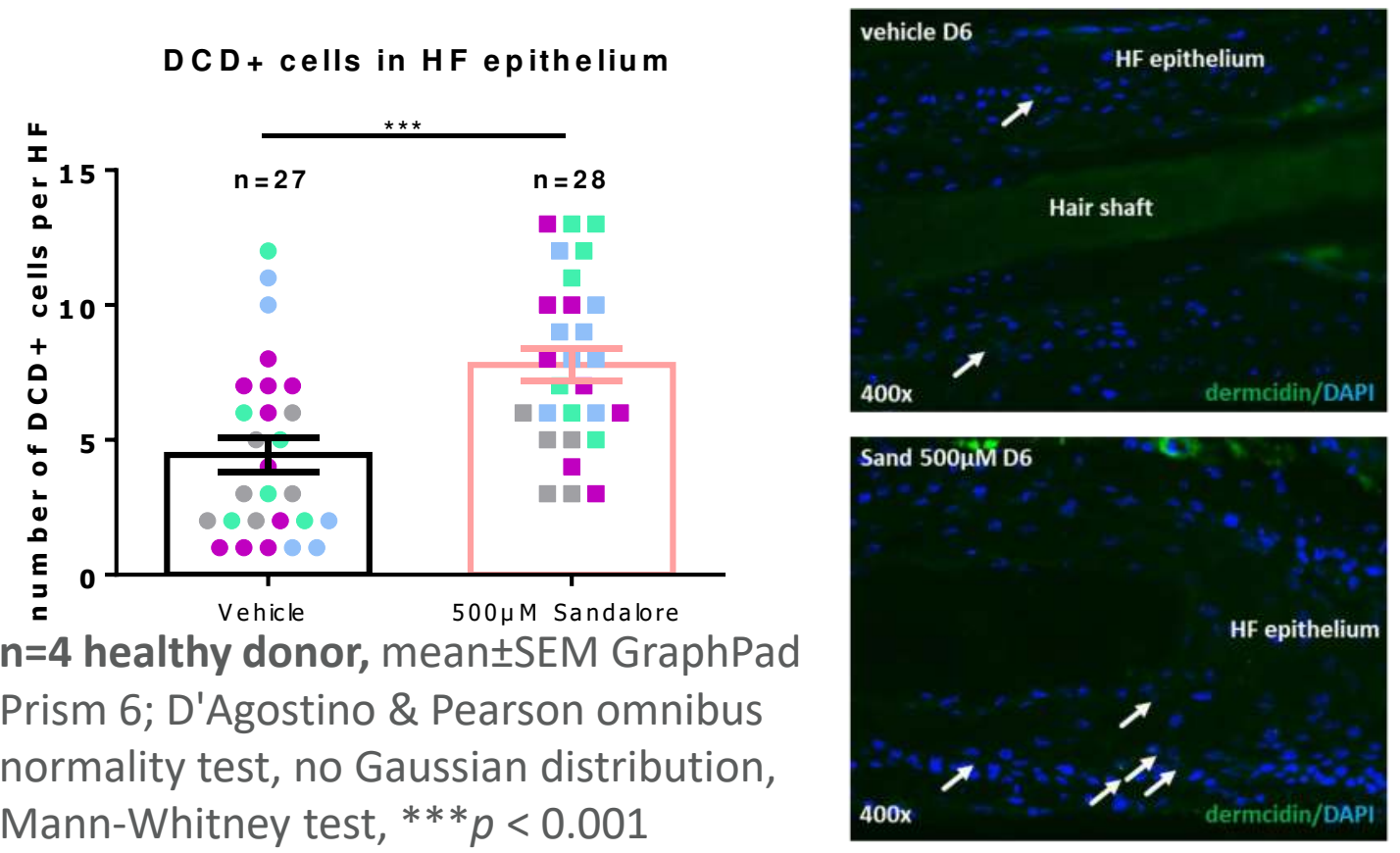

DCD positive spots were counted in the HF epithelium

3) Sandalore ${ }^{\circledR}$ reduces microbial contamination in microdissected HF ex vivo in the absence of antibiotics
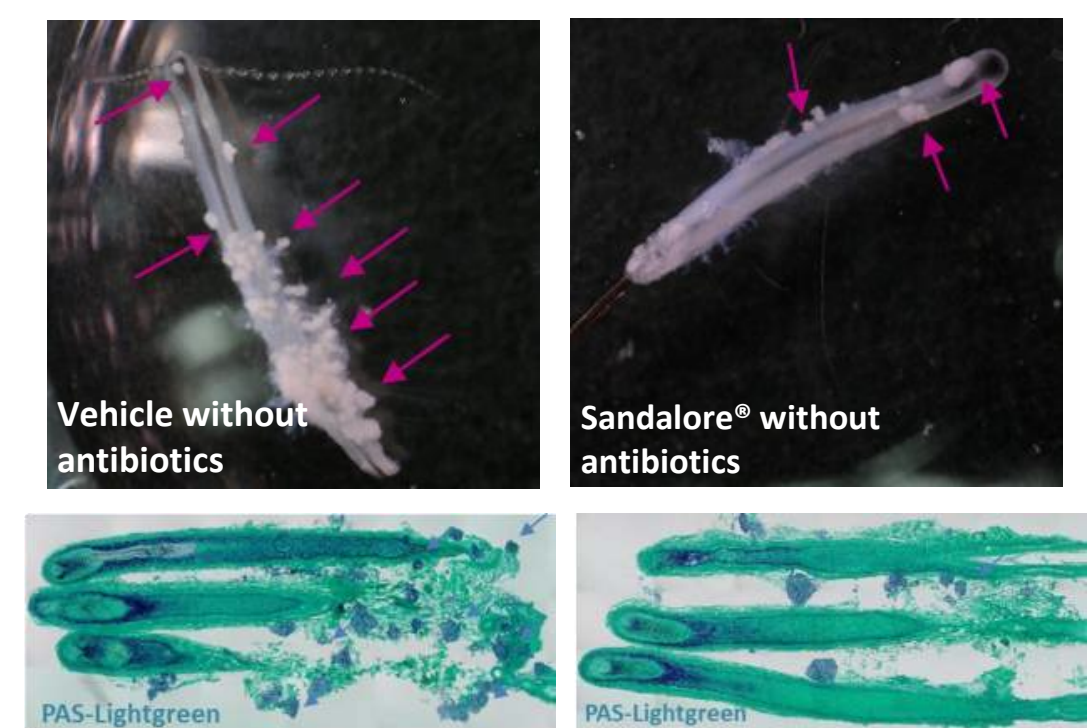

PAS staining in combination with Lightgreen can detect different sorts of fungi and mucous yeast films. In another experiment with amputated HF, treatment with Sandalore ${ }^{\circledR}$ prevented bacterial contaminations (data not shown).

$\mathrm{n}=7-8 \mathrm{HF} / \mathrm{group}$ from one healthy donor, mean \pm SEM GraphPad Prism 6; D'Agostino \& Pearson omnibus normality test, no Gaussian distribution, Mann-Whitney test, $* * * p<0.001$ compared to vehicle respectively; number and mean area of the colonies was quantified per hair follicle

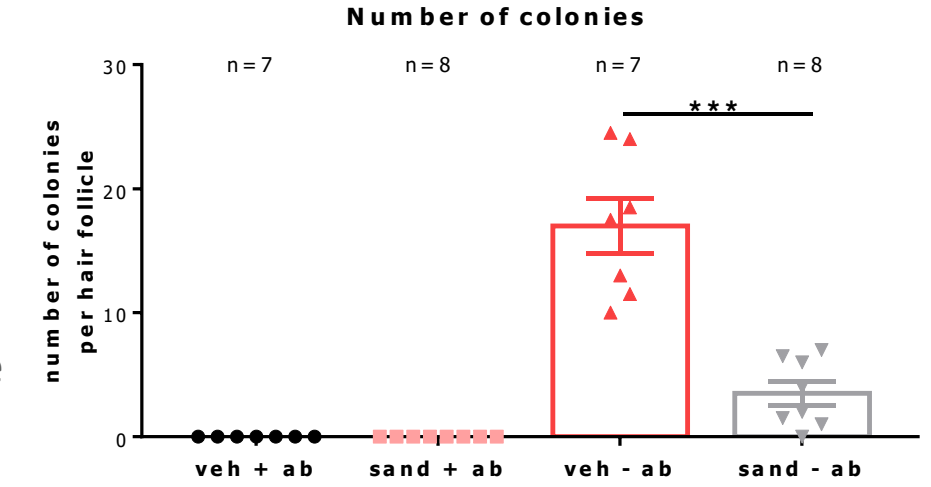

\section{CONCLUSION \& OUTLOOK}

DCD exerts broad antimicrobial activity against pathogenic microorganisms by interacting with bacterial membrane phospholipids and forming pores in the cell membrane. Our data show that stimulation of OR2AT4 by Sandalore ${ }^{\circledR}$ increases the antimicrobial activity of human HFs by stimulating DCD production. Thus, the data suggest that "olfactotherapy" of hair follicle diseases characterized by major microbial imbalances such as acne vulgaris or bacterial folliculitis, with selected odorants is a realistic possibility.
\end{abstract}

